\title{
Generalizing the Hypergraph Laplacian via a Diffusion Process with Mediators
}

\author{
T-H. Hubert Chan* Zhibin Liang*
}

\begin{abstract}
In a recent breakthrough STOC 2015 paper, a continuous diffusion process was considered on hypergraphs (which has been refined in a recent JACM 2018 paper) to define a Laplacian operator, whose spectral properties satisfy the celebrated Cheeger's inequality. However, one peculiar aspect of this diffusion process is that each hyperedge directs flow only from vertices with the maximum density to those with the minimum density, while ignoring vertices having strict in-beween densities.

In this work, we consider a generalized diffusion process, in which vertices in a hyperedge can act as mediators to receive flow from vertices with maximum density and deliver flow to those with minimum density. We show that the resulting Laplacian operator still has a second eigenvalue satsifying the Cheeger's inequality.

Our generalized diffusion model shows that there is a family of operators whose spectral properties are related to hypergraph conductance, and provides a powerful tool to enhance the development of spectral hypergraph theory. Moreover, since every vertex can participate in the new diffusion model at every instant, this can potentially have wider practical applications.
\end{abstract}

\footnotetext{
*Department of Computer Science, the University of Hong Kong. hubert@cs.hku.hk,
} liangzbeconnect.hku.hk 


\section{Introduction}

Spectral graph theory, and specifically, the well-known Cheeger's inequality give a relationship between the edge expansion properties of a graph and the eigenvalues of some appropriately defined matrix [1, 2]. Loosely speaking, for a given graph, its edge expansion or conductance gives a lower bound on the ratio of the number of edges leaving a subset $S$ of vertices to the sum of vertex degrees in $S$. It is natural that graph conductance is studied in the context of graph partitioning or clustering [9, 17, 18], whose goal is to minimize the weight of edges crossing different clusters with respect to intra-cluster edges. The reader can refer to the standard references [5, 8] for an introduction to spectral graph theory.

Recent Generalization to Hypergraphs. In an edge-weighted hypergraph $H=(V, E, w)$, an edge $e \in E$ is a non-empty subset of $V$. The edges have positive weights indicated by $w: E \rightarrow \mathbb{R}_{+}$. The weight of each vertex $v \in V$ is its weighted degree $w_{v}:=\sum_{e \in E: v \in e} w_{e}$. A subset $S$ of vertices has weight $w(S):=\sum_{v \in S} w_{v}$, and the edges it cuts is $\partial S:=\{e \in E: e$ intersects both $S$ and $V \backslash S\}$.

The conductance of $S \subseteq V$ is defined as $\phi(S):=\frac{w(\partial S)}{w(S)}$. The conductance of $H$ is defined as:

$$
\phi_{H}:=\min _{\emptyset \subsetneq S \subsetneq V} \max \{\phi(S), \phi(V \backslash S)\} .
$$

Until recently, it was an open problem to define a spectral model for hypergraphs. In a breakthrough STOC 2015 paper, Louis [13] considered a continuous diffusion process on hypergraphs (which has been refined in a recent JACM paper [3]), and defined an operator $\mathrm{L}_{w} f:=-\frac{d f}{d t}$, where $f \in \mathbb{R}^{V}$ is some appropriate vector associated with the diffusion process. As in classical spectral graph theory, $\mathrm{L}_{w}$ has non-negative eigenvalues, and the all-ones vector 1 is an eigenvector with eigenvalue 0 . Moreover, the operator $\mathrm{L}_{w}$ has a second eigenvalue $\gamma_{2}$, and the Cheeger's inequality can be recovered 1 for hypergraphs:

$$
\frac{\gamma_{2}}{2} \leq \phi_{H} \leq 2 \sqrt{\gamma_{2}}
$$

Limitation of the Existing Diffusion Model [3, 13]. Suppose at some instant, each vertex has some measure that is given by a measure vector $\varphi \in \mathbb{R}^{V}$. A corresponding density vector $f \in \mathbb{R}^{V}$ is defined by $f_{u}:=\frac{\varphi_{u}}{w_{u}}$, for each $u \in V$. Then, at this instant, each edge $e \in E$ will cause measure to flow from vertices $S_{e}(f):=\operatorname{argmax}_{s \in e} f_{s}$ having the maximum density to vertices $I_{e}(f):=\operatorname{argmin}_{i \in e} f_{i}$ having the minimum density, at a rate of $w_{e} \cdot \max _{s, i \in e}\left(f_{s}-f_{i}\right)$. Observe that there can be more than one vertex achieving the maximum or the minimum density in an edge, and a vertex can be involved with multiple number of edges. As shown in [3], it is non-trivial to determine the net rate of incoming measure for each vertex.

One peculiar aspect of this diffusion process is that each edge $e$ only concerns its vertices having the maximum or the minimum density, and ignores the vertices having strict in-between densities. Even though this diffusion process leads to a theoretical treatment of spectral hypergraph properties, its practical use is somehow limited, because it would be considered more natural if vertices having intermediate densities in an edge also take part in the diffusion process.

For instance, in a recent work on semi-supervised learning on hypergraphs [20], the diffusion operator is used to construct an update vector that changes only the solution values of vertices attaining the maximum or the minimum in hyperedges. Therefore, we consider the following open problem in this work:

Is there a diffusion process on hypergraphs that involves all vertices in every edge at every instant such that the resulting operator still retains desirable spectral properties?

\subsection{Our Contribution and Results.}

Generalized Diffusion Process with Mediators. We consider a diffusion process where for each edge $e$, a vertex $j \in e$ can act as a mediator that receives flow from vertices in $S_{e}(f)$ and delivers flow to $I_{e}(f)$. Formally, we denote $[e]:=e \cup\{0\}$, where 0 is a special index that does not refer to any

\footnotetext{
${ }^{1}$ In fact, as shown in this work, a stronger upper bound holds: $\phi_{H} \leq \sqrt{2 \gamma_{2}}$.
} 
vertex. Each edge $e$ is equipped with non-negative constants $\left(\beta_{j}^{e}: j \in[e]\right)$ such that $\sum_{j \in[e]} \beta_{j}^{e}=1$. Intuitively, for $j=0, \beta_{0}^{e}$ refers to the effect of flow going directly from $S_{e}(f)$ to $I_{e}(f)$; for each vertex $j \in e, \beta_{j}^{e}$ refers to the significance of $j$ as a mediator between $S_{e}(f)$ and $I_{e}(f)$. The complete description of the diffusion rules is in Definition 3.1. Here are some interesting special cases captured by the new diffusion model.

- For each $e \in E, \beta_{0}^{e}=1$. This is the existing model in [3, 13].

- For each $e \in E$, there is some $j_{e} \in e$ such that $\beta_{j_{e}}^{e}=1$, i.e., each edge has one special vertex that acts as its mediator who regulates all flow within the edge.

- For each $e \in E$, for each $j \in e, \beta_{j}^{e}=\frac{1}{|e|}$, i.e., every vertex in an edge are equally important as mediators.

Theorem 1.1 (Recovering Cheeger's Inequality via Diffusion Process with Mediators) Given a hypergraph $H=(V, E, w)$ and mediator constants $\left(\beta_{j}^{e}: e \in E, j \in[e]\right)$, the diffusion process in Definition 3.1 defines an operator $\mathrm{L}_{w} f:=-\frac{d f}{d t}$ that has a second eigenvalue $\gamma_{2}$ satisfying $\frac{\gamma_{2}}{2} \leq \phi_{H} \leq 2 \sqrt{\gamma_{2}}$, where $\phi_{H}$ is the hypergraph conductance defined in (1.1).

Impacts of New Diffusion Model. Our generalized diffusion model shows that there is a family of operators whose spectral properties are related to hypergraph conductance. On the theoretical aspect, this provides a powerful tool to enhance the development of spectral hypergraph theory.

On the practical aspect, as mentioned earlier, in the context of semi-supervised learning [7, 20], the following minimization convex program is considered: the objective function is $Q(f):=\left\langle f, \mathrm{~L}_{w} f\right\rangle_{w}$, and the $f$ values of labeled vertices are fixed. For an iterative method to solve the convex program, our new diffusion model can possibly lead to an update vector that modifies every coordinate in the current solution, thereby potentially improving the performance of the solver.

\subsection{Related Work}

Other Works on Diffusion Process and Spectral Graph Theory. Apart from the most related aforementioned works [3, 13] that we have already mentioned, similar diffusion models (without mediators) have been considered for directed normal graphs [19] and directed hypergraphs [4] to define operators whose spectral properties are analyzed.

Higher-Order Cheeger Inequalities. For normal graphs, Cheeger-like inequalities to relate higher-order spectral properties with multi-way edge expansion have been investigated [10, 11, 12, 14, 15, 16]. On the other hand, for hypergraphs, the higher-order spectral properties of the diffusion operator are still unknown. However, Cheeger-like inequalities have been derived in terms of the discrepancy ratio [3], but not related to the spectral properties of the diffusion operator.

\section{Preliminaries}

We consider an edge-weighted hypergraph $H=(V, E, w)$. Without loss of generality, we assume that the weight $w_{i}:=\sum_{e \in E: i \in e} w_{e}$ of each vertex $i \in V$ is positive, since any vertex with zero weight can be removed. We use $\mathrm{W} \in \mathbb{R}^{V \times V}$ to denote the diagonal matrix whose $(i, i)$-th entry is the vertex weight $w_{i}$; we let $I_{n}$ denote the identity matrix.

We use $\mathbb{R}^{V}$ to denote the set of column vectors. Given $f \in \mathbb{R}^{V}$, we use $f_{u}$ or $f(u)$ to indicate the coordinate corresponding to $u \in V$. We use $A^{\top}$ to denote the transpose of a matrix $A$.

We use $\mathbf{1} \in \mathbb{R}^{V}$ to denote the vector having 1 in every coordinate. For a vector $x \in \mathbb{R}^{V}$, we define its support as the set of coordinates at which $x$ is non-zero, i.e. $\operatorname{supp}(x):=\left\{i: x_{i} \neq 0\right\}$.

We use $\chi_{S} \in\{0,1\}^{V}$ to denote the indicator vector of the set $S \subset V$, i.e., $\chi_{S}(v)=1$ iff $v \in S$.

Recall that the conductance $\phi_{H}$ of a hypergraph $H$ is defined in (1.1). We drop the subscript whenever the hypergraph is clear from the context. 
Generalized Quadratic Form. For each edge $e \in E$, we denote $[e]:=e \cup\{0\}$, where 0 is a special index that does not correspond to any vertex. Then, each edge $e$ is associated with non-negative constants $\left(\beta_{j}^{e}: j \in[e]\right)$ such that $\sum_{j \in[e]} \beta_{j}^{e}=1$. The generalized quadratic form is defined for each $f \in \mathbb{R}^{V}$ as:

$$
\mathrm{Q}(f):=\sum_{e \in E} w_{e}\left\{\beta_{0}^{e} \max _{s, i \in e}\left(f_{s}-f_{i}\right)^{2}+\sum_{j \in e} \beta_{j}^{e}\left[\left(\max _{s \in e} f_{s}-f_{j}\right)^{2}+\left(f_{j}-\min _{i \in e} f_{i}\right)^{2}\right]\right\}
$$

For each non-zero $f \in \mathbb{R}^{V}$, its discrepancy ratio is defined as $\mathrm{D}_{w}(f):=\frac{\mathrm{Q}(f)}{\sum_{u \in V} w_{u} f_{u}^{2}}$.

Remark. Observe that for each $S \subseteq V$, the corresponding indicator vector $\chi(S) \in\{0,1\}^{V}$ satisfies $\mathrm{Q}(\chi(S))=w(\partial S)$. Hence, we have $\mathrm{D}_{w}(\chi(S))=\phi(S)$.

Special Case. We denote $Q^{0}(f):=\sum_{e \in E} w_{e} \max _{s, i \in e}\left(f_{s}-f_{i}\right)^{2}$ for the case when $\beta_{0}^{e}=1$ for all $e$, which was considered in [3]. As we shall see later, for $j \in e$, the weight $\beta_{j}^{e}$ denotes the significance of vertex $j$ as a "mediator" in the diffusion process to direct measure from vertices of maximum density to those with minimum density. As in [3], we consider three isomorphic spaces as follows.

Density Space. This is the space associated with the quadratic form Q. For $f, g \in \mathbb{R}^{V}$, the inner product is defined as $\langle f, g\rangle_{w}:=f^{\top} \mathrm{W} g$, and the associated norm is $\|f\|_{w}:=\sqrt{\langle f, f\rangle_{w}}$. We use $f \perp_{w} g$ to denote $\langle f, g\rangle_{w}=0$.

Normalized Space. Given $f \in \mathbb{R}^{V}$ in the density space, the corresponding vector in the normalized space is $x:=\mathrm{W}^{\frac{1}{2}} f$. The normalized discrepancy ratio is $\mathcal{D}(x):=\mathrm{D}_{w}\left(\mathrm{~W}^{-\frac{1}{2}} x\right)=\mathrm{D}_{w}(f)$.

In the normalized space, the usual $\ell_{2}$ inner product and norm are used. Observe that if $x$ and $y$ are the corresponding normalized vectors for $f$ and $g$ in the density space, then $\langle x, y\rangle=\langle f, g\rangle_{w}$.

Towards Cheeger's Inquality. Using the inequality $a^{2}+b^{2} \leq(a+b)^{2} \leq 2\left(a^{2}+b^{2}\right)$ for non-negative $a$ and $b$, we conclude that $\mathrm{Q}(f) \leq \mathrm{Q}^{0}(f) \leq 2 \mathrm{Q}(f)$ for all $f \in \mathbb{R}^{V}$. This immediately gives a partial result of Theorem 1.1 .

Lemma 2.1 (Cheeger's Inequality for Quadratic Form) Suppose $\gamma_{2}:=\min _{\mathbf{0} \neq f \perp_{w} \mathbf{1}} \frac{Q(f)}{\|f\|_{w}^{2}}$. Then, we have $\frac{\gamma_{2}}{2} \leq \phi_{H} \leq 2 \sqrt{\gamma_{2}}$, where $\phi_{H}$ is the hypergraph conductance defined in (1.1).

Proof: Denote $\gamma_{2}^{0}:=\min _{\mathbf{0} \neq f \perp_{w} \mathbf{1}} \frac{\mathrm{Q}^{0}(f)}{\|f\|_{w}^{2}}$. Then, the result from [3] and an improved upper bound in Appendix B gives: $\frac{\gamma_{2}^{0}}{2} \leq \phi_{H} \leq \sqrt{2 \gamma_{2}^{0}}$. Finally, $\mathrm{Q} \leq \mathrm{Q}^{0} \leq 2 \mathrm{Q}$ implies that $\gamma_{2} \leq \gamma_{2}^{0} \leq 2 \gamma_{2}$. Hence, the result follows.

Goal of This Paper. In view of Lemma2.1, the most technical part of the paper is to define an operatot $\mathrm{L}_{w}: \mathbb{R}^{V} \rightarrow \mathbb{R}^{V}$ such that $\left\langle f, \mathrm{~L}_{w} f\right\rangle_{w}=\mathrm{Q}(f)$, and show that $\gamma_{2}$ defined in Lemma 2.1 is indeed an eigenvalue of $\mathrm{L}_{w}$. To achieve this, we shall consider a diffusion process in the following measure space.

Measure Space. Given a density vector $f \in \mathbb{R}^{V}$, multiplying each coordinate with its corresponding weight gives the measure vector $\varphi:=\mathrm{W} f$. Observe that a vector in the measure space can have negative coordinates. We do not consider inner product explicitly in this space, and so there is no special notation for it.

Transformation between Different Spaces. We use the Roman letter $f$ for vectors in the density space, $x$ for vectors in the normalized space, and Greek letter $\varphi$ for vectors in the measure space. Observe that an operator defined on one space induces operators on the other two spaces. For instance, if $L$ is an operator defined on the measure space, then $L_{w}:=W^{-1} L W$ is the corresponding operator on the density space and $\mathcal{L}:=W^{-\frac{1}{2}} \mathrm{LW} \mathrm{W}^{\frac{1}{2}}$ is the one on the normalized space. Moreover, all three operators have the same eigenvalues. Recall that the Rayleigh quotients are defined as $\mathrm{R}_{w}(f):=\frac{\left\langle f, \mathrm{~L}_{w} f\right\rangle_{w}}{\langle f, f\rangle_{w}}$ and $\mathcal{R}(x):=\frac{\langle x, \mathcal{L} x\rangle}{\langle x, x\rangle}$. For $\mathrm{W}^{\frac{1}{2}} f=x$, we have $\mathrm{R}_{w}(f)=\mathcal{R}(x)$.

\footnotetext{
${ }^{2}$ In the literature, the weighted Laplacian is actually $\mathrm{WL}_{w}$ in our notation. Hence, to avoid confusion, we restrict the term Laplacian to the normalized space.
} 


\section{Diffusion Process with Mediators}

Intuition. Given an edge-weighted hypergraph $H=(V, E, w)$, suppose at some instant, each vertex has some measure given by the vector $\varphi \in \mathbb{R}^{V}$, whose corresponding density vector is $f=\mathrm{W}^{-1} \varphi$. The idea of a diffusion process is that within each edge $e \in E$, measure should flow from vertices with higher densities to those with lower densities, and the rate of flow has a positive correlation with the difference in densities and the strength of the edge $e$ given by $w_{e}$. If the diffusion process is well-defined, then an operator on the density space can be defined as $\mathrm{L}_{w} f:=-\frac{d f}{d t}$. This induces the Laplacian operator $\mathcal{L}:=\mathrm{W}^{\frac{1}{2}} \mathrm{~L}_{w} \mathrm{~W}^{-\frac{1}{2}}$ on the normalized space.

In previous work [3], within an edge, measure only flows from vertices $S_{e}(f):=\operatorname{argmax}_{s \in e} f_{s} \subseteq e$ having the maximum density to those $I_{e}(f):=\operatorname{argmin}_{i \in e} f_{i}$ having minimum densities, where the rate of flow is $w_{e} \cdot \max _{s, i \in e}\left(f_{s}-f_{i}\right)$. If all $f_{u}$ 's for an edge $e$ are equal, then we use the convention that $I_{e}(f)=S_{e}(f)=e$. Note that vertices $j \in e \backslash\left(S_{e}(f) \cup I_{e}(f)\right)$ with strict in-between densities do not participate due to edge $e$ at this instant.

Generalized Diffusion Process with Mediators. In some applications as mentioned in Section 1, it might be more natural if every vertex in an edge $e$ plays some role in diverting flow from $S_{e}(f)$ to $I_{e}(f)$. In our new diffusion model, each edge $e$ is associated with constants $\left(\beta_{j}^{e}: j \in[e]\right)$ such that $\sum_{j \in[e]} \beta_{j}^{e}=1$.

Here, 0 is a special index and the parameter $\beta_{0}^{e}$ corresponds to the significance of measure flowing directly from $S_{e}(f)$ to $I_{e}(f)$. For $j \in e, \beta_{j}^{e}$ indicates the significance of vertex $j$ as a "mediator" to receive measure from $S_{e}(f)$ and deliver measure to $I_{e}(f)$. The formal rules are given as follows.

Definition 3.1 (Rules of Diffusion Process) Suppose at some instant the system is in a state given by the density vector $f \in \mathbb{R}^{V}$, with measure vector $\varphi=\mathrm{W} f$. Then, at this instant, measure is transferred between vertices according to the following rules. For $u \in e$ and $j \in[e]$, the pair $(e, j)$ imposes some rules on the diffusion process; let $\varphi_{u}^{\prime}(e, j)$ be the net rate of measure flowing into vertex $u$ due to the pair $(e, j)$.

$R(0)$ For each vertex $u \in V$, the density changes according to the net rate of incoming measure divided by its weight:

$$
w_{u} \frac{d f_{u}}{d t}=\varphi_{u}^{\prime}:=\sum_{e \in E: u \in e} \sum_{j \in[e]} \varphi_{u}^{\prime}(e, j) .
$$

$R(1)$ We have $\varphi_{u}^{\prime}(e, j)<0$ and $u \neq j$ implies that $u \in S_{e}(f)$. Similarly, $\varphi_{u}^{\prime}(e, j)>0$ and $u \neq j$ implies that $u \in I_{e}(f)$.

$R(2)$ Each edge $e \in E$ and $j \in[e]$, the rates of flow satisfy the following. For $j=0$, the rate of flow from $S_{e}(f)$ to $I_{e}(f)$ due to $(e, 0)$ is:

$$
-\sum_{u \in S_{e}(f)} \varphi_{u}^{\prime}(e, 0)=w_{e} \cdot \beta_{0}^{e} \cdot \max _{s, i \in e}\left(f_{s}-f_{i}\right)=\sum_{u \in I_{e}(f)} \varphi_{u}^{\prime}(e, 0) \text {. }
$$

For $j \in e$, the rate of flow from $S_{e}(f)$ to $j$ due to $(e, j)$ is:

$$
-\sum_{u \in S_{e}(f)} \varphi_{u}^{\prime}(e, j)=w_{e} \cdot \beta_{j}^{e} \cdot\left(\max _{s \in e} f_{s}-f_{j}\right)
$$

the rate of flow from $j$ to $I_{e}(f)$ due to $(e, j)$ is:

$$
\sum_{u \in I_{e}(f)} \varphi_{u}^{\prime}(e, j)=w_{e} \cdot \beta_{j}^{e} \cdot\left(f_{j}-\min _{i \in e} f_{i}\right) .
$$

Then the net rate of flow received by $j$ due to $(e, j)$ is:

$$
w_{e} \cdot \beta_{j}^{e} \cdot\left(\max _{s \in e} f_{s}+\min _{i \in e} f_{i}-2 f_{j}\right)=\varphi_{j}^{\prime}(e, j) .
$$

Existence of Diffusion Process. The diffusion rules in Definition 3.1 are much more complicated than those in [3]. It is not immediately obvious whether such a process is well-defined. However, the techniques in [4] can be employed. Intuitively, by repeatedly applying the procedure described in Section 4, all higher-order derivatives of the density vector can be determined, which induce an equivalence relation on $V$ such that vertices in the same equivalence class will have the same density in infinitesimal time. This means the hypergraph can be reduced to a simple graph, in which the diffusion process is known to be well-defined. However, to argue this formally is non-trivial, and the reader can refer to the details in [4].

As in [3], if we define an operator using the diffusion process in Definition 3.1, then the resulting 
Rayleigh quotient coincides with the discrepancy ratio. The proof of the following lemma is deferred to Appendix A.

Lemma 3.2 (Rayleigh Quotient Coincides with Discrepancy Ratio) Suppose $\mathrm{L}_{w}$ on the density space is defined as $\mathrm{L}_{w} f:=-\frac{d f}{d t}$ by the rules in Definition 3.1. Then, the Rayleigh quotient associated with $\mathrm{L}_{w}$ satisfies that for any $f$ in the density space, $\mathrm{R}_{w}(f)=\mathrm{D}_{w}(f)$. By considering the isomorphic normalized space, we have for each $x, \mathcal{R}(x)=\mathcal{D}(x)$.

\section{Computing the First Order Derivative in the Diffusion Process}

In Section 3 , we define a diffusion process, whose purpose is to define an operator $\mathrm{L}_{w} f:=-\frac{d f}{d t}$, where $f \in \mathbb{R}^{V}$ is in the density space. In this section, we show that the diffusion rules uniquely determine the first order derivative vector $\frac{d f}{d t}$; moreover, we give an algorithm to compute it.

Infinitesimal Considerations. In Definition 3.1, if a vertex $u$ is losing measure due to the pair $(e, j)$ and $u \neq j$, then $u$ must be in $S_{e}(f)$. However, $u$ must also continue to stay in $S_{e}(f)$ in infinitesimal time; otherwise, if $u$ is about to leave $S_{e}(f)$, then $u$ should no longer lose measure due to $(e, j)$. Hence, the vertex $u$ should have the maximum first-order derivative of $f_{u}$ among vertices in $S_{e}(f)$. A similar rule should hold when $u$ is gaining measure due to $(e, j)$ and $u \neq j$. This is formalized as the first-order variant of $(R 1)$ :

Rule (R3) First-Order Derivative Constraints:

If $\varphi_{u}^{\prime}(e, j)<0$ and $u \neq j$, then $u \in \operatorname{argmax}_{s \in S_{e}(f)} \frac{d f_{s}}{d t}$.

If $\varphi_{u}^{\prime}(e, j)>0$ and $u \neq j$, then $u \in \operatorname{argmin}_{i \in I_{e}(f)} \frac{d f_{i}}{d t}$.

Considering Each Equivalence Class $U$ Independently. As in [3], we consider the equivalence relation induced by $f \in \mathbb{R}^{V}$, where two vertices $u$ and $v$ are in the same equivalence class iff $f_{u}=f_{v}$. For vertices in some equivalence class $U$, their current $f$ values are the same, but their values could be about to be separated because their first derivatives might be different.

Subset with the Largest First Derivative: Densest Subset. Suppose $X \subseteq U$ are the vertices having the largest derivative in $U$. Then, these vertices should receive or contribute rates of measure in each of the following cases.

1. The subset $X$ receives measure due to edges $I_{X}:=\left\{e \in E: I_{e}(f) \subseteq X\right\}$, because the corresponding vertices in $X$ continue to have minimum $f$ values in these edges; we let $c_{e}^{I} \geq 0$ be the rate of measure received by $I_{e}(f)$ due to $(e, j)$ for $j \notin I_{e}(f)$.

2. The subset $X$ contributes measure due to edges $S_{X}:=\left\{e \in E: S_{e}(f) \cap X \neq \emptyset\right\}$, because the corresponding vertices in $X$ continue to have maximum $f$ values in these edges; we let $c_{e}^{S} \geq 0$ be the rate of measure delivered by $S_{e}(f)$ due to $(e, j)$ for $j \notin S_{e}(f)$.

3. Each $j \in X$ receives or contributes measure due to all $(e, j)$ 's such that $e \in E$ and $j \in e$; we let $c_{j} \in \mathbb{R}$ be the net rate of measure received by vertex $j$ due to $(e, j)$ for all $e \in E$ such that $j \in e$.

Hence, the net rate of measure received by $X$ is

$$
\mathfrak{C}(X):=\sum_{e \in I_{X}} c_{e}^{I}-\sum_{e \in S_{X}} c_{e}^{S}+\sum_{j \in X} c_{j}
$$

Therefore, given an instance $\left(U, I_{U}, S_{U}\right)$, the problem is to find a maximal subset $P \subseteq U$ with the largest density $\delta(P):=\frac{\mathfrak{c}(P)}{w(P)}$, which will be the $\frac{d f}{d t}$ values for the vertices in $P$. For the remaining vertices in $U$, the sub-instance $\left(U \backslash P, I_{U} \backslash I_{P}, S_{U} \backslash S_{P}\right)$ is solved recursively. The procedure and the precise parameters are given in Fig. 4.1. Efficient algorithms for this densest subset problem are described in [6, 3]. 
Given a hypergraph $H=(V, E, w)$ and a vector $f \in \mathbb{R}^{V}$ in the density space, define an equivalence relation on $V$ such that $u$ and $v$ are in the same equivalence class iff $f_{u}=f_{v}$. We consider each such equivalence class $U \subseteq V$ and define the $r=\frac{d f}{d t}$ values for vertices in $U$ as follows.

1. Denote $E_{U}:=\left\{e \in E: U \cap\left[I_{e}(f) \cup S_{e}(f)\right] \neq \emptyset\right\}$.

For $e \in E$, define

$c_{e}^{I}:=w_{e} \cdot\left[\beta_{0}^{e} \cdot \max _{s, i \in e}\left(f_{s}-f_{i}\right)+\sum_{j \in e} \beta_{j}^{e} \cdot\left(f_{j}-\min _{i \in e} f_{i}\right)\right]$,

$c_{e}^{S}:=w_{e} \cdot\left[\beta_{0}^{e} \cdot \max _{s, i \in e}\left(f_{s}-f_{i}\right)+\sum_{j \in e} \beta_{j}^{e} \cdot\left(\max _{s \in e} f_{s}-f_{j}\right)\right] ;$

for $j \in V$, define $c_{j}:=\sum_{e \in E: j \in e} \beta_{j}^{e} \cdot w_{e} \cdot\left(\max _{s \in e} f_{s}+\min _{i \in e} f_{i}-2 f_{j}\right)$.

For $X \subseteq U$, define $I_{X}:=\left\{e \in E_{U}: I_{e}(f) \subseteq X\right\}, S_{X}:=\left\{e \in E_{U}: S_{e}(f) \cap X \neq \emptyset\right\}$.

Denote $\mathfrak{C}(X):=\sum_{e \in I_{X}} c_{e}^{I}-\sum_{e \in S_{X}} c_{e}^{S}+\sum_{j \in X} c_{j}$ and $\delta(X):=\frac{\mathfrak{C}(X)}{w(X)}$.

2. Find $P \subseteq U$ such that $\delta(P)$ is maximized. For all $u \in P$, set $r_{u}:=\delta(P)$.

3. Recursively, find the $r$ values for the remaining vertices in $U^{\prime}:=U \backslash P$ using $E_{U^{\prime}}:=E_{U} \backslash$ $\left(I_{P} \cup S_{P}\right)$.

Figure 4.1: Procedure to compute $r=\frac{d f}{d t}$

The next lemma shows that the procedure in Fig. 4.1 returns a vector $r \in \mathbb{R}^{V}$ that coincides with the first-order derivative $\frac{d f}{d t}$ of the density vector obeying rules (R0) to (R3). This implies that these rules uniquely determine the first-order derivative. Given $f \in \mathbb{R}^{V}$ and $r=\frac{d f}{d t}$, we denote $r_{S}(e):=$ $\max _{u \in S_{e}(f)} r_{u}$ and $r_{I}(e):=\min _{u \in I_{e}(f)} r_{u}$.

Lemma 4.1 (Densest Subset Problem Determines First-Order Deriative) Given a density vector $f \in$ $\mathbb{R}^{V}$, rules (RO) to (R3) uniquely determine $r=\frac{d f}{d t} \in \mathbb{R}^{V}$, which can be found by the procedure described in Fig. 4.1 Moreover, $\sum_{e \in E} c_{e}^{I} \cdot r_{I}(e)-\sum_{e \in E} c_{e}^{S} \cdot r_{S}(e)+\sum_{j \in V} c_{j} \cdot r_{j}=\sum_{u \in V} \varphi_{u}^{\prime} r_{u}=\|r\|_{w}^{2}$.

Proof: Using the same approach as in [3], we consider each equivalence class $U$ in Fig. 4.1, where all vertices in a class have the same $f$ values.

For each such equivalence class $U \subset V$, define $I_{U}:=\left\{e \in E: U \cap I_{e}(f) \neq \emptyset\right\}, S_{U}:=\{e \in E$ : $\left.U \cap S_{e}(f) \neq \emptyset\right\}$. Notice that each $e$ can only be in exactly one of $I_{U}$ and $S_{U}$.

Considering Each Equivalence Class $U$. Suppose $T$ is the set of vertices within $U$ that have the maximum first-order derivative $r=\frac{d f}{d t}$. It suffices to show that $T$ is the maximal densest subset in the densest subset instance $\left(U, I_{U} \cup S_{U}\right)$ defined in Fig. 4.1 .

Because of rule (R3), the rate of net measure received by $T$ is $\mathfrak{C}(T)$. Hence, all vertices $u \in T$ have $r_{u}=\frac{\mathfrak{C}(T)}{w(T)}$.

Next, suppose $P$ is the maximal densest subset found in Fig. 4.1. Observe that the net rate of measure entering $P$ is at least $\mathfrak{C}(P)$. Hence, there exists some vertex $v \in P$ such that $\frac{\mathfrak{C}(P)}{w(P)} \leq r_{v} \leq \frac{\mathfrak{C}(T)}{w(T)}$, where the last inequality follows from the definition of $T$.

Since $P$ is the maximal densest subset, it follows that in the above inequality, actually all equalities hold and all vertices in $P$ have the same $r$ value. In general, the maximal densest subset contains all densest subsets, and it follows that $T \subseteq P$. Since all vertices in $P$ have the maximum $r$ value within $U$, we conclude that $P=T$.

Recursive Argument. Hence, it follows that the set $T$ can be uniquely identified in Fig. 4.1 as the set of vertices having maximum $r$ values, which is also the unique maximal densest subset. Then, the argument can be applied recursively for the smaller instance with $U^{\prime}:=U \backslash T, I_{U^{\prime}}:=I_{U} \backslash I_{T}, S_{U^{\prime}}:=S_{U} \backslash S_{T}$. 
Claim. $\sum_{e \in E} c_{e}^{I} \cdot r_{I}(e)-\sum_{e \in E} c_{e}^{S} \cdot r_{S}(e)+\sum_{j \in V} c_{j} \cdot r_{j}=\sum_{u \in V} \varphi_{u}^{\prime} r_{u}=\|r\|_{w}^{2}$.

Consider some $T$ defined above with $\delta:=\delta(T)=r_{u}$, for $u \in T$.

Observe that

$$
\begin{aligned}
\sum_{u \in T} \varphi_{u}^{\prime} r_{u} & =\left(\sum_{e \in I_{T}} c_{e}^{I}-\sum_{e \in S_{T}} c_{e}^{S}+\sum_{j \in T} c_{j}\right) \cdot \delta \\
& =\sum_{e \in I_{T}} c_{e}^{I} \cdot \min _{i \in I_{e}} r_{i}-\sum_{e \in S_{T}} c_{e}^{S} \cdot \max _{s \in S_{e}} r_{s}+\sum_{j \in T} c_{j} \cdot r_{j}
\end{aligned}
$$

where the last equality is due to rule (R3).

Observe that every $u \in V$ will be in exactly one such $T$, and every $e \in E$ will be accounted for exactly once in each of $I_{T}$ and $S_{T}$, ranging over all $T$ 's. Hence, summing over all $T$ 's gives the result.

\section{Spectral Properties of Laplacian}

A classical result in spectral graph theory is that for a 2-graph whose edge weights are given by the adjacency matrix $A$, the parameter $\gamma_{2}:=\min _{\mathbf{0} \neq x \perp \mathrm{W}^{\frac{1}{2}} \mathbf{1}} \mathcal{D}(x)$ is an eigenvalue of the normalized Laplacian $\mathcal{L}:=\mathrm{I}_{n}-\mathrm{W}^{-\frac{1}{2}} A \mathrm{~W}^{-\frac{1}{2}}$, where a corresponding minimizer $x_{2}$ is an eigenvector of $\mathcal{L}$. Observe that $\gamma_{2}$ is also an eigenvalue on the operator $\mathrm{L}_{w}:=\mathrm{I}_{n}-\mathrm{W}^{-1} A$ induced on the density space.

In this section, we generalize the result to hypergraphs. Observe that any result for the normalized space has an equivalent counterpart in the density space, and vice versa.

Theorem 5.1 (Eigenvalue of Hypergraph Laplacian) For a hypergraph with edge weights $w$, there exists a normalized Laplacian $\mathcal{L}$ such that the normalized discrepancy ratio $\mathcal{D}(x)$ coincides with the corresponding Rayleigh quotient $\mathcal{R}(x)$. Moreover, the parameter $\gamma_{2}:=\min _{\mathbf{0} \neq x \perp \mathrm{W}^{\frac{1}{2}} \mathbf{1}} \mathcal{D}(x)$ is an eigenvalue of $\mathcal{L}$, where any minimizer $x_{2}$ is a corresponding eigenvector.

Before proving Theorem 5.1, we first consider the spectral properties of the normalized Laplacian $\mathcal{L}$ induced by the diffusion process defined in Section 4 .

Lemma 5.2 (First-Order Derivatives) Consider the diffusion process satisfying rules (R0) to (R3) on the measure space with $\varphi \in \mathbb{R}^{V}$, which corresponds to $f=\mathrm{W}^{-1} \varphi$ in the density space. Suppose $\mathrm{L}_{w}$ is the induced operator on the density space such that $\frac{d f}{d t}=-\mathrm{L}_{w} f$. Then, we have the following derivatives.

1. $\frac{d\|f\|_{w}^{2}}{d t}=-2\left\langle f, \mathrm{~L}_{w} f\right\rangle_{w}$.

2. $\frac{d\left\langle f, \mathrm{~L}_{w} f\right\rangle_{w}}{d t}=-2\left\|\mathrm{~L}_{w} f\right\|_{w}^{2}$.

3. Suppose $\mathrm{R}_{w}(f)$ is the Rayleigh quotient with respect to the operator $\mathrm{L}_{w}$ on the density space. Then, for $f \neq \mathbf{0}, \frac{d \mathrm{R}_{w}(f)}{d t}=-\frac{2}{\|f\|_{w}^{4}} \cdot\left(\|f\|_{w}^{2} \cdot\left\|\mathrm{L}_{w} f\right\|_{w}^{2}-\left\langle f, \mathrm{~L}_{w} f\right\rangle_{w}^{2}\right) \leq 0$, by the Cauchy-Schwarz inequality on the $\langle\cdot, \cdot\rangle_{w}$ inner product, where equality holds iff $\mathrm{L}_{w} f \in \operatorname{span}(f)$.

By considering a transformation to the normalized space, for any $x \neq 0, \frac{d \mathcal{R}(x)}{d t} \leq 0$, where equality holds iff $\mathcal{L} x \in \operatorname{span}(x)$.

Proof: For the first statement, $\frac{d\|f\|_{w}^{2}}{d t}=2\left\langle f, \frac{d f}{d t}\right\rangle_{w}=-2\left\langle f, \mathrm{~L}_{w} f\right\rangle_{w}$.

For the second statement, from the proof of Lemma 3.2 we have

$$
\left\langle f, \mathrm{~L}_{w} f\right\rangle_{w}=\sum_{e \in E} w_{e}\left\{\beta_{0}^{e} \max _{s, i \in e}\left(f_{s}-f_{i}\right)^{2}+\sum_{j \in e} \beta_{j}^{e}\left[\left(\max _{s \in e} f_{s}-f_{j}\right)^{2}+\left(f_{j}-\min _{i \in e} f_{i}\right)^{2}\right]\right\} .
$$


Hence, by the Envelope Theorem,

$$
\begin{aligned}
\frac{d\left\langle f, \mathrm{~L}_{w} f\right\rangle_{w}}{d t}= & 2 \sum_{e \in E} w_{e}\left[\beta_{0}^{e} \max _{s, i \in e}\left(f_{s}-f_{i}\right)\left(\max _{s \in S_{e}} \frac{d f_{s}}{d t}-\min _{i \in I_{e}} \frac{d f_{i}}{d t}\right)\right] \\
& +\sum_{j \in e} \beta_{j}^{e}\left(\max _{s \in e} f_{s}-f_{j}\right)\left(\max _{s \in S_{e}} \frac{d f_{s}}{d t}-\frac{d f_{j}}{d t}\right) \\
& \left.+\sum_{j \in e} \beta_{j}^{e}\left(f_{j}-\min _{i \in e} f_{i}\right)\left(\frac{d f_{j}}{d t}-\min _{i \in I_{e}} \frac{d f_{i}}{d t}\right)\right] \\
= & 2 \sum_{e \in E} w_{e}\left\{\left[\beta_{0}^{e} \max _{s, i \in e}\left(f_{s}-f_{i}\right)+\sum_{j \in e} \beta_{j}^{e}\left(\max _{s \in e} f_{s}-f_{j}\right)\right] \max _{s \in S_{e}} \frac{d f_{s}}{d t}\right. \\
& -\left[\beta_{0}^{e} \max _{s, i \in e}\left(f_{s}-f_{i}\right)+\sum_{j \in e} \beta_{j}^{e}\left(f_{j}-\min _{i \in e} f_{i}\right)\right] \min _{i \in I_{e}} \frac{d f_{i}}{d t} \\
& \left.+\sum_{j \in e} \beta_{j}^{e}\left(2 f_{j}-\max _{s \in e} f_{s}-\min _{i \in e} f_{i}\right) \frac{d f_{j}}{d t}\right\} . \\
= & 2\left(\sum_{e \in E} c_{e}^{I} \cdot \max _{s \in S_{e}} r_{s}-\sum_{e \in E} c_{e}^{S} \cdot \max _{i \in I_{e}} r_{i}-\sum_{j \in V} c_{j} \cdot r_{j}\right)
\end{aligned}
$$

where $c_{e}^{I}, c_{e}^{S}, c_{j}$ are defined in Fig. 4.1, From Lemma 4.1, this equals $-2\|r\|_{w}^{2}=-2\left\|\mathrm{~L}_{w} f\right\|_{w}^{2}$.

Finally, for the third statement, we have $\frac{d}{d t} \frac{\left\langle f, \mathrm{~L}_{w} f\right\rangle_{w}}{\langle f, f\rangle_{w}}=\frac{1}{\|f\|_{w}^{4}}\left(\|f\|_{w}^{2} \cdot \frac{d\left\langle f, \mathrm{~L}_{w} f\right\rangle_{w}}{d t}-\left\langle f, \mathrm{~L}_{w} f\right\rangle_{w} \cdot \frac{d\|f\|_{w}^{2}}{d t}\right)=$ $-\frac{2}{\|f\|_{w}^{4}} \cdot\left(\|f\|_{w}^{2} \cdot\left\|\mathrm{L}_{w} f\right\|_{w}^{2}-\left\langle f, \mathrm{~L}_{w} f\right\rangle_{w}^{2}\right)$, where the last equality follows from the first two statements.

We next prove some properties of the normalized Laplacian $\mathcal{L}$ with respect to orthogonal projection in the normalized space.

Lemma 5.3 (Laplacian and Orthogonal Projection) Suppose $\mathcal{L}$ is the normalized Laplacian. Moreover, denote $x_{1}:=\mathrm{W}^{\frac{1}{2}} 1$, and let $\Pi$ denote the orthogonal projection into the subspace that is orthogonal to $x_{1}$. Then, for all $x$, we have the following:

1. $\mathcal{L}(x) \perp x_{1}$,

2. $\langle x, \mathcal{L} x\rangle=\langle\Pi x, \mathcal{L} \Pi x\rangle$.

3. For all real numbers $a$ and $b, \mathcal{L}\left(a x_{1}+b x\right)=b \mathcal{L}(x)$.

Proof: For the first statement, observe that since the diffusion process is defined on a closed system, the total measure given by $\sum_{u \in V} \varphi_{u}$ does not change. Therefore, $0=\left\langle\mathbf{1}, \frac{d \varphi}{d t}\right\rangle=\left\langle\mathrm{W}^{\frac{1}{2}} \mathbf{1}, \frac{d x}{d t}\right\rangle$, which implies that $\mathcal{L} x=-\frac{d x}{d t} \perp x_{1}$.

For the second statement, observe that from Lemma 3.2, we have $\langle x, \mathcal{L} x\rangle=\sum_{e \in E} w_{e}\left\{\beta_{0}^{e} \max _{s, i \in e}\left(\frac{x_{s}}{\sqrt{w_{s}}}-\right.\right.$ $\left.\left.\frac{x_{i}}{\sqrt{w_{i}}}\right)^{2}+\sum_{j \in e} \beta_{j}^{e}\left[\left(\max _{s \in e} \frac{x_{s}}{\sqrt{w_{s}}}-\frac{x_{j}}{\sqrt{w_{j}}}\right)^{2}+\left(\frac{x_{j}}{\sqrt{w_{j}}}-\min _{i \in e} \frac{x_{i}}{\sqrt{w_{i}}}\right)^{2}\right]\right\}=\left\langle\left(x+\alpha x_{1}\right), \mathcal{L}\left(x+\alpha x_{1}\right)\right\rangle$, where the last equality holds for all real numbers $\alpha$. Observe that $\Pi x=x+\alpha x_{1}$, for some suitable real $\alpha$.

For the third statement, it is more convenient to consider transformation into the density space $f=$ $\mathrm{W}^{-\frac{1}{2}} x$. It suffices to show that $\mathrm{L}_{w}(a \mathbf{1}+b f)=b \mathrm{~L}_{w}(f)$.

Observe that in the diffusion process, only pairwise difference in densities among vertices matters. Hence, we immediately have $\mathrm{L}_{w}(a \mathbf{1}+b f)=\mathrm{L}_{w}(b f)$.

For $b \geq 0$, observe that all the rates are scaled by the same factor $b$. Hence, we have $\mathrm{L}_{w}(b f)=b \mathrm{~L}_{w}(f)$.

Finally, if we reverse the sign of every coordinate of $f$, then the roles of $S_{e}(f)$ and $I_{e}(f)$ are switched. Moreover, the direction of every component of the measure flow is reversed with the same magnitude. Hence, $\mathrm{L}_{w}(-f)=-\mathrm{L}_{w}(f)$, and the result follows.

Proof of Theorem 5.1: This follows the same argument as in [3]. Suppose $\mathcal{L}$ is the normalized Laplacian induced by the diffusion process in Lemma 4.1. Let $\gamma_{2}:=\min _{\mathbf{0} \neq x \perp \mathrm{W}^{\frac{1}{2}} \mathbf{1}} \mathcal{R}(x)$ be attained by some minimizer $x_{2}$. We use the isomorphism between the three spaces: $\mathrm{W}^{-\frac{1}{2}} \varphi=x=\mathrm{W}^{\frac{1}{2}} f$.

The third statement of Lemma 5.2 can be formulated in terms of the normalized space, which states that $\frac{d \mathcal{R}(x)}{d t} \leq 0$, where equality holds iff $\mathcal{L} x \in \operatorname{span}(x)$. 
We claim that $\frac{d \mathcal{R}\left(x_{2}\right)}{d t}=0$. Otherwise, suppose $\frac{d \mathcal{R}\left(x_{2}\right)}{d t}<0$. From Lemma 5.3, we have $\frac{d x}{d t}=-\mathcal{L} x \perp$ $\mathrm{W}^{\frac{1}{2}} \mathbf{1}$. Hence, it follows that at this moment, the current normalized vector is at position $x_{2}$, and is moving towards the direction given by $x^{\prime}:=\left.\frac{d x}{d t}\right|_{x=x_{2}}$ such that $x^{\prime} \perp \mathrm{W}^{\frac{1}{2}} \mathbf{1}$, and $\left.\frac{d \mathcal{R}(x)}{d t}\right|_{x=x_{2}}<0$. Therefore, for sufficiently small $\epsilon>0$, it follows that $x_{2}^{\prime}:=x_{2}+\epsilon x^{\prime}$ is a non-zero vector such that $x_{2}^{\prime} \perp \mathrm{W}^{\frac{1}{2}} \mathbf{1}$ and $\mathcal{R}\left(x_{2}^{\prime}\right)<\mathcal{R}\left(x_{2}\right)=\gamma_{2}$, contradicting the definition of $x_{2}$.

Hence, it follows that $\frac{d \mathcal{R}\left(x_{2}\right)}{d t}=0$, which implies that $\mathcal{L} x_{2} \in \operatorname{span}\left(x_{2}\right)$. Since $\gamma_{2}=\mathcal{R}\left(x_{2}\right)=\frac{\left\langle x_{2}, \mathcal{L} x_{2}\right\rangle}{\left\langle x_{2}, x_{2}\right\rangle}$, it follows that $\mathcal{L} x_{2}=\gamma_{2} x_{2}$, as required.

\section{References}

[1] N. Alon. Eigenvalues and expanders. Combinatorica, 6(2):83-96, 1986.

[2] N. Alon and V. D. Milman. $\lambda 1$, isoperimetric inequalities for graphs, and superconcentrators. Journal of Combinatorial Theory, Series B, 38(1):73-88, 1985.

[3] T. H. Chan, A. Louis, Z. G. Tang, and C. Zhang. Spectral properties of hypergraph laplacian and approximation algorithms. J. ACM, 65(3):15:1-15:48, 2018.

[4] T. H. Chan, Z. G. Tang, X. Wu, and C. Zhang. Diffusion operator and spectral analysis for directed hypergraph laplacian. CoRR, abs/1711.01560, 2017.

[5] F. R. Chung. Spectral graph theory, volume 92. American Mathematical Soc., 1997.

[6] M. Danisch, T. H. Chan, and M. Sozio. Large scale density-friendly graph decomposition via convex programming. In $W W W$, pages 233-242. ACM, 2017.

[7] M. Hein, S. Setzer, L. Jost, and S. S. Rangapuram. The total variation on hypergraphs - learning on hypergraphs revisited. In NIPS, pages 2427-2435, 2013.

[8] S. Hoory, N. Linial, and A. Wigderson. Expander graphs and their applications. Bulletin of the American Mathematical Society, 43(4):439-561, 2006.

[9] R. Kannan, S. Vempala, and A. Vetta. On clusterings: Good, bad and spectral. J. ACM, 51(3):497$515,2004$.

[10] T. C. Kwok, L. C. Lau, and Y. T. Lee. Improved cheeger's inequality and analysis of local graph partitioning using vertex expansion and expansion profile. In SODA, pages 1848-1861. SIAM, 2016.

[11] T. C. Kwok, L. C. Lau, Y. T. Lee, S. O. Gharan, and L. Trevisan. Improved cheeger's inequality: analysis of spectral partitioning algorithms through higher order spectral gap. In STOC, pages 11-20. ACM, 2013.

[12] J. R. Lee, S. O. Gharan, and L. Trevisan. Multiway spectral partitioning and higher-order cheeger inequalities. Journal of the ACM (JACM), 61(6):37, 2014.

[13] A. Louis. Hypergraph markov operators, eigenvalues and approximation algorithms. In STOC, pages 713-722. ACM, 2015.

[14] A. Louis and K. Makarychev. Approximation algorithm for sparsest k-partitioning. In SODA, pages 1244-1255. SIAM, 2014.

[15] A. Louis, P. Raghavendra, P. Tetali, and S. Vempala. Algorithmic extensions of cheeger's inequality to higher eigenvalues and partitions. In APPROX-RANDOM, volume 6845 of Lecture Notes in Computer Science, pages 315-326. Springer, 2011. 
[16] A. Louis, P. Raghavendra, P. Tetali, and S. Vempala. Many sparse cuts via higher eigenvalues. In STOC, pages 1131-1140. ACM, 2012.

[17] K. Makarychev, Y. Makarychev, and A. Vijayaraghavan. Correlation clustering with noisy partial information. In COLT, volume 40 of JMLR Workshop and Conference Proceedings, pages 13211342. JMLR.org, 2015.

[18] R. Peng, H. Sun, and L. Zanetti. Partitioning well-clustered graphs: Spectral clustering works! In COLT, volume 40 of JMLR Workshop and Conference Proceedings, pages 1423-1455. JMLR.org, 2015.

[19] Y. Yoshida. Nonlinear laplacian for digraphs and its applications to network analysis. In Proceedings of the Ninth ACM International Conference on Web Search and Data Mining, pages 483-492. ACM, 2016.

[20] C. Zhang, S. Hu, Z. G. Tang, and T. H. Chan. Re-revisiting learning on hypergraphs: Confidence interval and subgradient method. In ICML, volume 70 of Proceedings of Machine Learning Research, pages 4026-4034. PMLR, 2017. 


\section{A Rayleigh Quotient Coincides with Discrepancy Ratio}

To prove Lemma 3.2, we first re-interpret the diffusion rules in Definition 3.1 by considering the interaction between every pair of nodes. Observe that the rules sometimes say that some measure is flow from one subset of vertices to another subset. Hence, at the moment, the exact pairwise interactions are not specified. In fact, we know that in general, the pairwise interactions are not uniquely determined. Fig. A.1 captures this non-deterministic nature of the pairwise interactions.

Given a hypergraph $H(V, E, w)$ and a density vector $f \in \mathbb{R}^{V}$, we analyze the pairwise interaction between vertices according to Definition 3.1 .

1. We shall describe constraints on the pairwise interaction by a symmetric matrix $A_{f} \in \mathbb{R}^{V \times V}$ such that for $u, v \in V$, the $(u, v)$-th entry $a_{u v}$ means that between $u$ and $v$, there is measure flowing from the vertex of higher density to that of lower density, at the rate of $a_{u v} \cdot\left|f_{u}-f_{v}\right|$.

Furthermore, we decompose $a_{u v}:=\sum_{e \in E} \sum_{j \in[e]} a_{u v}^{(e, j)}$, where the role of the pair $(e, j)$ is described in Definition 3.1, We use the convention that $a_{u v}^{(e, j)}=a_{v u}^{(e, j)}$.

2. Pairwise Interaction due to $(e, j)$. For each $e \in E$ and $j \in[e]$, we describe the pairwise interaction due to $(e, j)$.

For $j=0$, by considering the rate of flow from $S_{e}(f)$ to $I_{e}(f)$ due to $(e, 0)$, we infer that the partial weight $w_{e} \beta_{0}^{e}$ is somehow distributed among $(s, i) \in S_{e}(f) \times I_{e}(f)$. In other words, we have the constraint:

$\sum_{(s, i) \in S_{e}(f) \times I_{e}(f)} a_{s i}^{(e, 0)}=w_{e} \beta_{0}^{e}$.

For $j \in e$, by considering the rate of flow from $S_{e}(f)$ to $j$ due to $(e, j)$, we have the constraint: $\sum_{s \in S_{e}(f)} a_{s j}^{(e, j)}=w_{e} \beta_{j}^{e}$.

Similarly, by considering the rate of flow from $j$ to $I_{e}(f)$ due to $(e, j)$, we have the constraint:

$\sum_{i \in I_{e}(f)} a_{j i}^{(e, j)}=w_{e} \beta_{j}^{e}$.

For the remaining vertex pairs $(u, v)$ that are not involved with the pair $(e, j)$, we assign $a_{u v}^{(e, j)}=$ 0 .

3. In conclusion, the interaction matrix $A_{f}$ satisfies the following. For $u \neq v, A_{f}(u, v)=$ $\sum_{w_{u} \in E} \sum_{j \in[e]} a_{u v}^{(e, j)}$; moreover, for each $u \in V$, the row of $A_{f}$ corresponding to $u$ sums to

Then, the diffusion process is described by $\mathrm{W} \frac{d f}{d t}=\frac{d \varphi}{d t}=\left(A_{f} \mathrm{~W}^{-1}-\mathrm{I}_{n}\right) \varphi$. Therefore, the resulting Laplacian operators satisfies $\mathrm{L}(\varphi)=\left(\mathrm{I}_{n}-A_{f} \mathrm{~W}^{-1}\right) \varphi$ for the measure space and $\mathrm{L}_{w}(f)=\left(\mathrm{I}_{n}-\right.$ $\left.\mathrm{W}^{-1} A_{f}\right) f$ for the density space.

Figure A.1: Constraints on Pairwise Interactions

Proof of Lemma 3.2: It suffices to show that

$$
\left\langle f, \mathrm{~L}_{w} f\right\rangle_{w}=\sum_{e \in E} w_{e}\left\{\beta_{0}^{e} \max _{s, i \in e}\left(f_{s}-f_{i}\right)^{2}+\sum_{j \in e} \beta_{j}^{e}\left[\left(\max _{s \in e} f_{s}-f_{j}\right)^{2}+\left(f_{j}-\min _{i \in e} f_{i}\right)^{2}\right]\right\} .
$$

Recall that $\varphi=\mathrm{W} f$, and $\mathrm{L}_{w}=\mathrm{I}_{n}-\mathrm{W}^{-1} A_{f}$, where $A_{f}$ satisfies the constaints in Fig. A.1. 
Hence, it follows that

$$
\begin{aligned}
\left\langle f, \mathrm{~L}_{w} f\right\rangle_{w} & =f^{\top}\left(\mathrm{W}-A_{f}\right) f=\sum_{u v \in\left(\begin{array}{c}
V \\
2
\end{array}\right)} a_{u v}\left(f_{u}-f_{v}\right)^{2}=\sum_{u v \in\left(\begin{array}{c}
V \\
2
\end{array}\right)} \sum_{e \in E} \sum_{j \in[e]} a_{u v}^{(e, j)}\left(f_{u}-f_{v}\right)^{2} \\
& =\sum_{u v \in\left(\begin{array}{c}
V \\
2
\end{array}\right)} \sum_{e \in E}\left[a_{u v}^{(e, 0)}\left(f_{u}-f_{v}\right)^{2}+\sum_{j \in e} a_{u v}^{(e, j)}\left(f_{u}-f_{v}\right)^{2}\right]
\end{aligned}
$$

For the first term, we have

$$
\begin{aligned}
& \sum_{u v \in\left(\begin{array}{l}
V \\
2
\end{array}\right)} \sum_{e \in E} a_{u v}^{(e, 0)}\left(f_{u}-f_{v}\right)^{2} \\
= & \sum_{u v \in\left(\begin{array}{c}
V \\
2
\end{array}\right)} \sum_{e \in E:\{u v, v u\} \cap S_{e} \times I_{e} \neq \emptyset} a_{u v}^{(e, 0)}\left(f_{u}-f_{v}\right)^{2} \\
= & \sum_{e \in E} \sum_{s i \in\left(\begin{array}{c}
e \\
2
\end{array}\right):\{s i, i s\} \cap S_{e} \times I_{e} \neq \emptyset} a_{s i}^{(e, 0)}\left(f_{s}-f_{i}\right)^{2} \\
= & \sum_{e \in E} \sum_{s i \in S_{e} \times I_{e}} a_{s i}^{(e, 0)}\left(f_{s}-f_{i}\right)^{2} \\
= & \sum_{e \in E} w_{e} \beta_{0}^{e} \max _{s, i \in e}\left(f_{s}-f_{i}\right)^{2}
\end{aligned}
$$

For the second term, we have

$$
\begin{aligned}
& \sum_{u v \in\left(\begin{array}{c}
V \\
2
\end{array}\right)} \sum_{e \in E} \sum_{j \in e} a_{u v}^{(e, j)}\left(f_{u}-f_{v}\right)^{2} \\
= & \sum_{u v \in\left(\begin{array}{c}
V \\
2
\end{array}\right)} \sum_{e \in E}\left[\sum_{j \in e:\{u v, v u\} \cap S_{e} \times j \neq \emptyset} a_{u v}^{(e, j)}\left(f_{u}-f_{v}\right)^{2}+\sum_{j \in e:\{u v, v u\} \cap j \times I_{e} \neq \emptyset} a_{u v}^{(e, j)}\left(f_{u}-f_{v}\right)^{2}\right] \\
= & \sum_{e \in E} \sum_{s i \in\left(\begin{array}{l}
e \\
2
\end{array}\right)}\left[\sum_{j \in e:\{s i, i s\} \cap S_{e} \times j \neq \emptyset} a_{s i}^{(e, j)}\left(f_{s}-f_{i}\right)^{2}+\sum_{j \in e:\{s i, i s\} \cap j \times I_{e} \neq \emptyset} a_{s i}^{(e, j)}\left(f_{s}-f_{i}\right)^{2}\right] \\
= & \sum_{e \in E} \sum_{j \in e}\left[\sum_{s \in S_{e}} a_{s j}^{(e, j)}\left(f_{s}-f_{j}\right)^{2}+\sum_{i \in I_{e}} a_{j i}^{(e, j)}\left(f_{j}-f_{i}\right)^{2}\right] \\
= & \sum_{e \in E} \sum_{j \in e}\left[w_{e} \beta_{j}^{e}\left(\max _{s \in e} f_{s}-f_{j}\right)^{2}+w_{e} \beta_{j}^{e}\left(f_{j}-\min _{i \in e} f_{i}\right)^{2}\right] \\
= & \sum_{e \in E} w_{e} \sum_{j \in e} \beta_{j}^{e}\left[\left(\max _{s \in e} f_{s}-f_{j}\right)^{2}+\left(f_{j}-\min _{i \in e} f_{i}\right)^{2}\right]
\end{aligned}
$$

Thus, we conclude

$$
\begin{aligned}
\left\langle f, \mathrm{~L}_{w} f\right\rangle_{w} & =\sum_{u v \in\left(\begin{array}{c}
V \\
2
\end{array}\right)} \sum_{e \in E}\left[a_{u v}^{(e, 0)}\left(f_{u}-f_{v}\right)^{2}+\sum_{j \in e} a_{u v}^{(e, j)}\left(f_{u}-f_{v}\right)^{2}\right] \\
& =\sum_{e \in E} w_{e}\left\{\beta_{0}^{e} \max _{s, i \in e}\left(f_{s}-f_{i}\right)^{2}+\sum_{j \in e} \beta_{j}^{e}\left[\left(\max _{s \in e} f_{s}-f_{j}\right)^{2}+\left(f_{j}-\min _{i \in e} f_{i}\right)^{2}\right]\right\}
\end{aligned}
$$

as required. 


\section{B Improved Upper Bound for Hypergraph Cheeger Inequality}

Recall that we denote $\mathrm{Q}^{0}(f):=\sum_{e \in E} w_{e} \max _{s, i \in e}\left(f_{s}-f_{i}\right)^{2}$ for the special case in (2.1) when $\beta_{0}^{e}=1$ for all $e \in E$, and $\gamma_{2}^{0}:=\min _{\mathbf{0} \neq f \perp_{w} \mathbf{1}} \frac{\mathbf{Q}^{0}(f)}{\|f\|_{w}^{2}}$.

Theorem B.1 (Upper Bound for Hypergraph Cheeger Inequalities) Given an edge-weighted hypergraph $H$, we have:

$$
\phi_{H} \leq \min _{\mathbf{0} \neq f \perp_{w} \mathbf{1}} \sqrt{\frac{2 Q^{0}(f)}{\|f\|_{w}^{2}}}=\sqrt{2 \gamma_{2}^{0}},
$$

where $\phi_{H}$ is the hypergraph conductance defined in (1.1).

Proposition B.1 Given an edge-weighted hypergraph $H=(V, E, w)$ and a non-zero vector $f \in \mathbb{R}^{V}$ such that $f \perp_{w} \mathbf{1}$, there exists a set $S \subseteq \operatorname{supp}(f)$ such that

$$
\phi(S) \leq \sqrt{\frac{2 Q^{0}(f)}{\|f\|_{w}^{2}}} .
$$

Proof: Without loss of generality, suppose $-1 \leq f_{u} \leq 1$ for all $u \in V$ since we can scale $f$ if not. Let $t$ be a random variable that is uniformly distributed in $(0,1]$. Define $S_{t}:=\left\{u \in V: f_{u}^{2} \geq t\right\}$. Then $S_{t} \subseteq \operatorname{supp}(f)$ by definition. We consider the expected value of $w\left(S_{t}\right)$ and $w\left(\partial S_{t}\right)$.

$$
\begin{gathered}
\mathbb{E}\left[w\left(S_{t}\right)\right]=\sum_{u \in V} w_{u} \operatorname{Pr}\left[u \in S_{t}\right]=\sum_{u \in V} w_{u} \operatorname{Pr}\left[f_{u}^{2} \geq t\right]=\sum_{u \in V} w_{u} f_{u}^{2} . \\
\mathbb{E}\left[w\left(\partial S_{t}\right)\right]=\sum_{e \in E} w_{e} \operatorname{Pr}\left[e \in \partial S_{t}\right]=\sum_{e \in E} w_{e} \operatorname{Pr}\left[\min _{i \in e} f_{i}^{2}<t \leq \max _{s \in e} f_{s}^{2}\right] \\
=\sum_{e \in E} w_{e}\left(\max _{s \in e} f_{s}^{2}-\min _{i \in e} f_{i}^{2}\right)=\sum_{e \in E} w_{e} \max _{s, i \in e}\left(f_{s}^{2}-f_{i}^{2}\right)=\sum_{e \in E} w_{e} \max _{s, i \in e}\left(\left|f_{s}\right|^{2}-\left|f_{i}\right|^{2}\right) \\
=\sum_{e \in E} w_{e} \max _{s, i \in e}\left(\left|f_{s}\right|-\left|f_{i}\right|\right)\left(\left|f_{s}\right|+\left|f_{i}\right|\right) \leq \sum_{e \in E} w_{e} \max _{s, i \in e}\left(\left|f_{s}\right|-\left|f_{i}\right|\right) \max _{u, v \in e}\left(\left|f_{u}\right|+\left|f_{v}\right|\right) \\
\leq \sum_{e \in E} w_{e} \max _{s, i \in e}\left(f_{s}-f_{i}\right) \cdot \max _{u, v \in e}\left(\left|f_{u}\right|+\left|f_{v}\right|\right)
\end{gathered}
$$

(By Cauchy-Schwarz inequality)

$$
\begin{aligned}
& \leq \sqrt{\sum_{e \in E} w_{e} \max _{s, i \in e}\left(f_{s}-f_{i}\right)^{2}} \cdot \sqrt{\sum_{e \in E} w_{e} \max _{u, v \in e}\left(\left|f_{u}\right|+\left|f_{v}\right|\right)^{2}} \\
& \leq \sqrt{\mathrm{Q}^{0}(f)} \cdot \sqrt{\sum_{e \in E} w_{e} \max _{u, v \in e} 2\left(f_{u}^{2}+f_{v}^{2}\right)} \leq \sqrt{\mathrm{Q}^{0}(f)} \cdot \sqrt{\sum_{e \in E} w_{e} \cdot 2 \sum_{u \in e} f_{u}^{2}} \\
& =\sqrt{\mathrm{Q}^{0}(f)} \cdot \sqrt{2 \sum_{u \in V} w_{u} f_{u}^{2}}=\sqrt{\frac{2 \mathrm{Q}^{0}(f)}{\|f\|_{w}^{2}}} \cdot \sum_{u \in V} w_{u} f_{u}^{2} .
\end{aligned}
$$

Thus, $\frac{\mathbb{E}\left[w\left(\partial S_{t}\right)\right]}{\mathbb{E}\left[w\left(S_{t}\right)\right]} \leq \sqrt{\frac{2 Q^{0}(f)}{\|f\|_{w}^{2}}}$, which means $\mathbb{E}\left[w\left(\partial S_{t}\right)-\sqrt{\frac{2 Q^{0}(f)}{\|f\|_{w}^{2}}} \cdot w\left(S_{t}\right)\right] \leq 0$.

Therefore, there exists a $t$ such that the induced $S_{t}$ satisfies $\phi\left(S_{t}\right)=\frac{w\left(\partial S_{t}\right)}{w\left(S_{t}\right)} \leq \sqrt{\frac{2 \mathrm{Q}^{0}(f)}{\|f\|_{w}^{2}}}$.

For any $g \in \mathbb{R}^{V}$, define $g_{+}, g_{-} \in \mathbb{R}^{V}$ such that $g_{+}(v)=\max \{0, g(v)\}$ and $g_{-}(v)=\min \{0, g(v)\}$ for $v \in V$. 
Proposition B.2 Given an edge-weighted hypergraph $H=(V, E, w)$ and a non-zero vector $f \in \mathbb{R}^{V}$ such that $f \perp_{w} \mathbf{1}$, let $g=f-c \mathbf{1}$ where $c$ is a constant such that $w\left(\operatorname{supp}\left(g_{+}\right)\right) \leq \frac{w(V)}{2}$ and $w\left(\operatorname{supp}\left(g_{-}\right)\right) \leq \frac{w(V)}{2}$. Then we have the following:

1. $\|g\|_{w}^{2} \geq\|f\|_{w}^{2}$

2. $\mathrm{Q}^{0}(g)=\mathrm{Q}^{0}(f)$.

3. $\min \left\{\frac{\mathrm{Q}^{0}\left(g_{+}\right)}{\left\|g_{+}\right\|_{w}^{2}}, \frac{\mathrm{Q}^{0}\left(g_{-}\right)}{\left\|g_{-}\right\|_{w}^{2}}\right\} \leq \frac{\mathrm{Q}^{0}(g)}{\|g\|_{w}^{2}} \leq \frac{\mathrm{Q}^{0}(f)}{\|f\|_{w}^{2}}$.

Proof: For the first statement, let $h(c)=\|g\|_{w}^{2}=\sum_{u \in V} w_{u} g(u)^{2}=\sum_{u \in V} w_{u}\left(f_{u}-c\right)^{2}$. Then we have $h^{\prime}(c)=\sum_{u \in V}\left(-2 w_{u} f_{u}+2 c w_{u}\right)=\sum_{u \in V} 2 c w_{u}$, since $f \perp_{w} \mathbf{1}$. We also have $h^{\prime \prime}(c)=$ $\sum_{u \in V} 2 w_{u}>0$. Thus, $h(c)$ is minimized when $h^{\prime}(c)=0$, i.e. $c=0$. Then $\|g\|_{w}^{2}=h(c) \geq h(0)=$ $\sum_{u \in V} w_{u} f_{u}^{2}=\|f\|_{w}^{2}$.

For the second statement, we have

$\mathrm{Q}^{0}(g)=\sum_{e \in E} w_{e} \max _{u, v \in e}[g(u)-g(v)]^{2}=\sum_{e \in E} w_{e} \max _{u, v \in e}[f(u)-f(v)]^{2}=\mathrm{Q}^{0}(f)$.

For the third statement, notice that $\|g\|_{w}^{2}=\sum_{u \in V} w_{u} g(u)^{2}=\sum_{u \in V} w_{u} g_{+}(u)^{2}+\sum_{u \in V} w_{u} g_{-}(u)^{2}=$ $\left\|g_{+}\right\|_{w}^{2}+\left\|g_{-}\right\|_{w}^{2}$.

We claim that $\mathrm{Q}^{0}(g) \geq \mathrm{Q}^{0}\left(g_{+}\right)+\mathrm{Q}^{0}\left(g_{-}\right)$since for all $e \in E$,

$\max _{u, v \in e}[g(u)-g(v)]^{2} \geq \max _{u, v \in e}\left[g_{+}(u)-g_{+}(v)\right]^{2}+\max _{u, v \in e}\left[g_{-}(u)-g_{-}(v)\right]^{2}$

by considering the following two cases:

1. $g(u)$ and $g(u)$ have the same sign. Then it must be either $\forall u \in e, g(u) \geq 0$ or $\forall u \in e, g(u) \leq$ 0 . Thus, one of $\max _{u, v \in e}\left[g_{+}(u)-g_{+}(v)\right]^{2}$ and $\max _{u, v \in e}\left[g_{-}(u)-g_{-}(v)\right]^{2}$ equals zero. Then $\max _{u, v \in e}[g(u)-g(v)]^{2}=\max _{u, v \in e}\left[g_{+}(u)-g_{+}(v)\right]^{2}+\max _{u, v \in e}\left[g_{-}(u)-g_{-}(v)\right]^{2}$.

2. $g(u)$ and $g(u)$ have the opposite signs. With out loss of generality, we assume $g(u)>g(v)$. Then we have $g_{+}(u)>0, g_{-}(v)<0$ and $g_{+}(v)=g_{-}(u)=0$. Thus,

$$
\begin{aligned}
\max _{u, v \in e}[g(u)-g(v)]^{2} & =\max _{u, v \in e}\left[g(u)^{2}-2 g(u) g(v)+g(v)^{2}\right] \\
& \geq \max _{u \in e: g(u) \geq 0} g(u)^{2}+\max _{v \in e: g(v) \leq 0} g(v)^{2} \\
& =\max _{u \in e} g_{+}(u)^{2}+\max _{v \in e} g_{-}(v)^{2} \\
& =\max _{u, v \in e}\left[g_{+}(u)-g_{+}(v)\right]^{2}+\max _{u, v \in e}\left[g_{-}(u)-g_{-}(v)\right]^{2} .
\end{aligned}
$$

Then by the first and second statements, we have

$$
\frac{\mathrm{Q}^{0}(f)}{\|f\|_{w}^{2}} \geq \frac{\mathrm{Q}^{0}(g)}{\|g\|_{w}^{2}} \geq \frac{\mathrm{Q}^{0}\left(g_{+}\right)+\mathrm{Q}^{0}\left(g_{-}\right)}{\left\|g_{+}\right\|_{w}^{2}+\left\|g_{-}\right\|_{w}^{2}} \geq \min \left\{\frac{\mathrm{Q}^{0}\left(g_{+}\right)}{\left\|g_{+}\right\|_{w}^{2}}, \frac{\mathrm{Q}^{0}\left(g_{-}\right)}{\left\|g_{-}\right\|_{w}^{2}}\right\} .
$$

Now we can prove Theorem B.1

Proof of Theorem B.1: By Proposition B.1, there exist $S_{t+} \subseteq \operatorname{supp}\left(g_{+}\right)$and $S_{t-} \subseteq \operatorname{supp}\left(g_{-}\right)$such that for any non-zero vector $f \in \mathbb{R}^{V}$ satisfying $f \perp_{w} \mathbf{1}$ :

$$
\min \left\{\phi\left(S_{t+}\right), \phi\left(S_{t-}\right)\right\} \leq \min \left\{\sqrt{\frac{2 Q^{0}\left(g_{+}\right)}{\left\|g_{+}\right\|_{w}^{2}}}, \sqrt{\frac{2 Q^{0}\left(g_{-}\right)}{\left\|g_{-}\right\|_{w}^{2}}}\right\} \leq \sqrt{\frac{2 Q^{0}(f)}{\|f\|_{w}^{2}}}
$$

where the last inequality follows from the third statement of Proposition B.2. Moreover, $w\left(S_{t+}\right) \leq \frac{w(V)}{2}$ and $w\left(S_{t-}\right) \leq \frac{w(V)}{2}$ by Proposition B.2. Thus, for any non-zero vector $f \in \mathbb{R}^{V}$ satisfying $f \perp_{w} \mathbf{1}$ :

$$
\phi_{H} \leq \min \left\{\phi\left(S_{t+}\right), \phi\left(S_{t-}\right)\right\} \leq \sqrt{\frac{2 Q^{0}(f)}{\|f\|_{w}^{2}}} .
$$

Then we have $\phi_{H} \leq \min _{\mathbf{0} \neq f \perp_{w} \mathbf{1}} \sqrt{\frac{2 Q^{0}(f)}{\|f\|_{w}^{2}}}=\sqrt{2 \gamma_{2}^{0}}$. 